\title{
As tecnologias de informação e comunicação na vida de idosos com sintomas de
} depressão: significado, experiências e relacionamentos

\author{
Adriano Pasqualotti; PGIE/UFRGS; pasqualotti@upf.br \\ Dante Augusto Couto Barone; PGIE/UFRGS e PPGC/UFRGS; barone@inf.ufrgs.br \\ Johannes Doll; PPGEDU/UFRGS; johannes.doll@ufrgs.br
}

Resumo: Neste artigo, analisa-se o significado das tecnologias de informação e comunicação (TIC). Avaliam-se as experiências e os relacionamentos com o uso desses dispositivos numa coorte de idosos com sintomas de depressão atendidos por programas de saúde. Para detecção da depressão, utilizou-se a versão brasileira da escala de depressão geriátrica (GDS-15). Por meio da aplicação do mini-exame do estado mental (MMSE), realizou-se um diagnóstico da presença ou ausência de déficits cognitivos. Foram selecionados 56 idosos com sessenta anos ou mais que obtiveram escore maior ou igual a cinco pontos na GDS-15. Com relação à GDS e ao MMSE, os resultados indicam a escolaridade relaciona-se com o nível de desempenho cognitivo, mas não com a depressão. Já com relação às TIC, os principais achados indicam que os idosos usam para atender às necessidades de divertimento e entretenimento. Além disso, as tecnologias podem tornar-se um fator impeditivo para o convívio pessoal, porque o conhecimento cria uma minoria prestigiada, afastando aqueles que desconhecem daqueles que conhecem, ou, ao contrário, porque o conhecimento amplia a sua utilização em detrimento dos relacionamentos pessoais.

Palavras-chave: tecnologias de informação e comunicação; idosos; escala de depressão geriátrica; mini exame do estado mental.

\section{The information and communication technologies in the lives of elderly with symptoms of depression: meaning, experience and relationships}

\begin{abstract}
In this article, analyzes the meaning of the information and communication technologies (ICT). Assess whether the experiences and relationships with the use of these devices in a cohort of elderly patients with symptoms of depression served by health programs. For detection of depression, used to the Brazilian version of the Geriatric Depression Scale (GDS-15). Through the application of Mini-Mental State Examination (MMSE), held a diagnosis of the presence or absence of cognitive deficits. Were selected 56 elderly people sixty years or more who obtained score greater than or equal to five points in the GDS-15. With regard to the GDS and the MMSE the results indicate the education relates to the level of cognitive performance, but not with depression. Now with respect to ICT, the main findings indicate that the elderly use to meet the needs of fun and entertainment. Moreover, the technology can become an agent blocking for personal interaction, because the knowledge creates a prestigious minority, easing those unaware of those who know or because the knowledge expands its use to the detriment of personal relationships.
\end{abstract}


Keywords: information and communication technologies; elderly; geriatric depression scale; mini mental state examination.

Introdução

Sabe-se que a interação mediada pelas TIC assume características diferentes das interações face a face (Berge e Collins, 1995; Lévy, 1999). Para compreender essa diferença, é necessário considerar a interação social no ciberespaço como elemento principal desse processo. Por exemplo, os conhecimentos disponibilizados em ambientes informatizados na web para as pessoas idosas poderiam auxiliá-las no combate à exclusão sofrida nessa fase, possibilitando-lhes, ao mesmo tempo, vivenciar o agora, sem desprezar as experiências e os sentimentos já vivenciados. Em outras palavras, mais de que uma ligação com o mundo, a web torna-se um lugar legítimo de socialização. A comunicação que se desenvolve através dos textos e imagens construídas colaborativamente por meio do uso de tecnologias digitais de interação no ciberespaço expressa os novos paradigmas informacionais que estão se desenvolvendo na era da sociedade em rede. Castells (2006) indica que a revolução tecnológica que está ocorrendo caracteriza-se pela aplicação de conhecimento disponibilizado na rede de uma maneira não centralizada, para geração de novos conhecimentos num processo de realimentação cíclica entre a inovação e o seu uso. Segundo Bretas (2001, p. 29), as interações que ocorrem nas redes telemáticas "se delinea dentro de um estatuto epistemológico em constituição". Conforme Rogoff e Lave (1984), o indivíduo vive numa comunidade em virtude do que tem em comum com os outros, o que é possibilitado pela comunicação, que é o meio pelo qual participa dessa comunidade. Dessa forma, os encontros e as mediações sociais no ciberespaço, propiciados por ferramentas cooperativas e colaborativas, viabilizam as trocas de idéias e conhecimentos, bem como permitem o crescimento social do indivíduo por meio da conexão com os demais sujeitos da rede. Nesse sentido, o uso do computador com uma ferramenta colaborativa para a criação de espaços de comunicação e interação torna-se extremamente importante.

De acordo Kachar (2000), para possibilitarmos aos idosos a apropriação do computador, há a necessidade de envolvermos nesse processo três aspectos articulados entre si: o operacional, a linguagem da máquina e a abordagem pedagógica. No primeiro, o idoso aprende a operar o computador; desenvolvendo a habilidade e a destreza visomotora com o mouse, as teclas e os recursos de hardware e software. No segundo aspecto linguagem da máquina - trabalha-se a semântica dos menus, a tradução da palavra, o contexto de origem e o conceito subjacente, isto é, a leitura, interpretação e compreensão da nova linguagem tecnológica da comunicação. Por último, na abordagem pedagógica, utiliza-se um aplicativo para a promoção da interação/comunicação e para a construção do conhecimento. Ao interagir com o computador, o idoso pode depurar o seu pensamento sobre uma situação-problema. A criação de um desenho ou a digitação e formatação de um texto são elaboradas até satisfazerem o usuário. De acordo com Valente (1993), aprender por meio da descoberta há uma construção e apropriação do aprendido pelo aprendiz que o modifica. Esse descobrir é também a descoberta de si próprio, sentindo-se capaz de atingir seu objetivo, revelando suas potencialidades individuais e singulares. O autor descreve um ciclo de interação com o computador, levando o sujeito a depurar o seu pensamento em relação à situação problema: descrição-execução-reflexão-depuração (Rocha, 1993). 
Material e Métodos

A pesquisa é um estudo transversal de cunho quanti-qualitativo desenvolvido no período entre setembro de 2005 a maio de 2006. O objetivo foi analisar os processos de apropriação e significação das TIC por com uma coorte de idosos com diagnóstico de depressão residente na zona urbana do município de Passo Fundo - RS, Brasil. A pesquisa foi aprovada pelo Comitê de Ética em Pesquisa da Universidade de Passo Fundo - RS, Brasil. Em observância às diretrizes do Conselho Nacional de Saúde (CNS) do Ministério da Saúde (MS) do Brasil, o estudo atende às diretrizes no que se refere ao consentimento, sigilo e anonimato, benefícios e propriedade intelectual (Brasil, 2003). Os sujeitos que participaram da pesquisa foram indicados por agentes de saúde que trabalham em programas de saúde do governo federal brasileiro - PSF: Programa da Família; PACS: Programa de Agentes Comunitários de Saúde. Foram utilizados os indicadores socioeconômicos e demográficos gênero, escolaridade, estado civil e renda familiar para caracterizar a coorte pesquisada (Parahyba, 1998; Neri, 2001). Para definir população idosa como o grupo de pessoas com sessenta anos ou mais, utilizou-se o critério cronológico definido na Assembléia Mundial sobre Envelhecimento (WHO, 1984; Paschoal, 2005). Para o diagnóstico de episódios depressivos, de acordo com os critérios definidos na ICD-10 e DSM-IV (APA, 1994; WHO, 2006), utilizou-se a GDS-15, adaptada de Yesavage et al. (1982) por Almeida e Almeida (1999). Para analisar a presença ou ausência de déficit cognitivo empregou-se o MMSE, adaptado de Folstein, Folstein e McHugh (1975). Para definir se um sujeito da amostra apresentou déficit cognitivo, levou-se em conta a escolaridade, faixa etária e escore obtido no MMSE: (i) menos de quatro anos de ensino escolar: (a) entre 60 a 69 anos e escore de até 22 pontos; (b) entre 70 a 79 anos e escore de até 20 pontos; (c) mais de 79 anos e escore de até 18 pontos; (ii) ensino fundamental e escore de até 22 pontos; (iii) nível médio ou superior e escore de até 23 pontos. Para os sujeitos classificados nas categorias ii e iii não se levaram em conta as faixas etárias. Para analisar as pontuações da GDS-15 e os escores totais do MMSE, utilizou-se a análise de variância (ANOVA), ao nível de significância de 0,05. Para analisar o significado das TIC na vivência de idosos com sintomas de depressão, adotou-se como suporte metodológico a análise de conteúdo proposta por Bardin (2004).

\section{Resultados e Discussões}

A idade dos participantes apresentou $\mathrm{M}=69,2$ e $\mathrm{DP}=6,4(\mathrm{IC}=[67,5 ; 71,0])$. A renda familiar mensal per capita, em Real (R\$), apresentou $\mathrm{M}=539,32$ e $\mathrm{DP}=286,91$ (IC = $[460,24 ; 618,40])$, sendo que $57 \%$ dos sujeitos dispunham de mais de um salário mínimo por mês para suprir as necessidades básicas de saúde, alimentação, transporte e lazer. A população de pessoas com sessenta anos ou mais da zona urbana do município de Passo Fundo atendidas nos programas PACS e PSF em outubro de 2006 totalizava 6.608, sendo 57\% mulheres. A Tabela 1 apresenta as estatísticas dos indicadores demográficos da coorte pesquisada. Os dados indicam uma alta heterogeneidade para gênero e escolaridade, porém os valores demonstraram uma diferença significativa ( $p \leq$ $0,05)$ apenas para o primeiro indicador, indício da segregação ocupacional e funcional que ocorre entre homens e mulheres na área de gerontologia.

Tabela 1. Indicadores demográficos da coorte que obteve escore maior ou igual a cinco pontos na GDS-15 $(n=56)$. 


\begin{tabular}{llccc}
\hline & \multicolumn{3}{c}{ Novas Tecnologias na Educação } \\
\cline { 2 - 3 } & & \multicolumn{3}{c}{} \\
\hline Indicadores & Atributos & $\mathrm{n}\left(\%_{\mathrm{O}}\right)$ & $\mathrm{N}\left(\%_{\mathrm{E}}\right)$ & $p$ \\
\hline Gênero & Mulher & $46(82,1)$ & $611423(57,4)$ & 0,000 \\
& Homem & $10(17,9)$ & $454061(42,6)$ & \\
Escolaridade & 4 anos ou + & $42(75,0)$ & $848114(79,6)$ & 0,211 \\
& $<$ de 4 anos & $14(25,0)$ & $217370(20,4)$ & \\
\hline
\end{tabular}

Nota: Para a definição de escolaridade, levou-se em conta o analfabetismo, o período de frequiência à escola e o nível de aprendizado necessário para ler e escrever uma frase em português com começo, meio e fim (4 anos ou +: Alfabetizado com quatro anos ou mais de estudo; < de 4 anos: Analfabeto ou que obteve menos de quatro anos de estudo). Para testar a adequabilidade do conjunto de dados observados com o modelo probabilístico esperado, utilizou-se o teste qui-quadrado de aderência. Tomou-se como referência à população residente no Rio Grande do Sul em 2000 (IBGE, 2000); n (\%o) $=$ Freqüência observada; $\mathrm{N}\left(\%_{\mathrm{E}}\right)=$ Freqüência esperada.

O tempo, em anos, dos sujeitos que eram casados ou viviam com parceiros apresentou $\mathrm{M}=35,8$ e $\mathrm{DP}=12,5(\mathrm{IC}=[30,6 ; 41,1]) ;$ já o tempo de viuvez apresentou $\mathrm{M}=13,9 \mathrm{e}$ $\mathrm{DP}=12,7(\mathrm{IC}=[8,6 ; 19,3]) . \mathrm{O}$ escore total no MMSE obteve $\mathrm{M}=22,1$ e $\mathrm{DP}=5,0$ (IC $=[20,7 ; 23,4])$. Os sujeitos com presença de déficit cognitivo obtiveram $\mathrm{M}=18,2 \mathrm{e} \mathrm{DP}$ $=4,5(\mathrm{IC}=[16,3 ; 20,1]) ;$ já os com ausência obtiveram $\mathrm{M}=24,9$ e $\mathrm{DP}=3,2(\mathrm{IC}=$ $[23,8 ; 26,1])$. A pontuação na GDS-15 obteve $\mathrm{M}=9,2$ e $\mathrm{DP}=2,4(\mathrm{IC}=[8,5 ; 9,8])$. A Tabela 2 apresenta os resultados da análise descritiva da pontuação da GDS-15 em relação ao déficit cognitivo (presença e ausência) e à escolaridade, e do escore no MMSE em relação à escolaridade (4 anos ou $+;<$ de 4 anos).

Tabela 2. Estatísticas da GDS-15 em relação ao déficit cognitivo e à escolaridade, e do MMSE em relação à escolaridade.

\begin{tabular}{|c|c|c|c|c|c|c|c|c|c|}
\hline \multirow{2}{*}{ Indicadores } & \multirow{2}{*}{ Atributos } & \multirow{2}{*}{$\mathrm{n}$} & \multirow{2}{*}{ M } & \multirow{2}{*}{ SD } & \multirow{2}{*}{$\mathrm{SE}$} & \multicolumn{2}{|c|}{$95 \%$ CIM } & \multirow{2}{*}{ MIN } & \multirow{2}{*}{ MAX } \\
\hline & & & & & & LB & UB & & \\
\hline \multicolumn{10}{|c|}{ GDS-15 em relação ao déficit cognitivo e à escolaridade } \\
\hline \multirow[t]{2}{*}{ Déficit cognitivo } & $\operatorname{Sim}$ & 24 & 9,1 & 2,6 & 0,5 & 8,0 & 10,2 & 5 & 13 \\
\hline & Não & 32 & 9,2 & 2,3 & 0,4 & 8,4 & 10,0 & 5 & 14 \\
\hline \multirow[t]{2}{*}{ Escolaridade } & 4 anos ou + & 42 & 9,0 & 2,5 & 0,4 & 8,2 & 9,8 & 5 & 14 \\
\hline & $<$ de 4 anos & 14 & 9,6 & 2,0 & 0,5 & 8,4 & 10,7 & 5 & 13 \\
\hline \multicolumn{10}{|c|}{ MMSE em relação à escolaridade } \\
\hline \multirow[t]{2}{*}{ Escolaridade } & 4 anos ou + & 42 & 23,9 & 3,8 & 0,6 & 22,7 & 25,1 & 10 & 30 \\
\hline & $<$ de 4 anos & 14 & 16,5 & 4,1 & 1,1 & 14,2 & 18,8 & 8 & 21 \\
\hline
\end{tabular}

$\mathrm{M}=$ Média; $\mathrm{SD}=$ Desvio padrão; $\mathrm{SE}=$ Erro padrão; $95 \%$ CIM = Intervalo de confiança da média; $\mathrm{LB}=$ Limite inferior; UB = Limite superior; MIN = Mínimo; MAX = Máximo.

O teste de normalidade de Kolmogorov-Smirnovn revelou que a pontuação na GDS-15 $(0,116 ; p=0,058)$ e escore no $\operatorname{MMSE}(0,115 ; p=0,067)$ tem distribuição normal. $\mathrm{O}$ teste de Levene indica uma igualdade tanto da pontuação na GDS-15 em relação ao déficit cognitivo $(0,705 ; p=0,405)$ e à escolaridade $(1,860 ; p=0,178)$, quanto do escore no MMSE em relação à escolaridade $(0,071 ; p=0,790)$. A não violação da rejeição da homocedasticidade $(p \leq 0,01)$ possibilitou o uso da one-way para a analise da média da pontuação na GDS-15 e do escore no MMSE. A análise da variância (ANOVA) do escore da GDS-15 não apresentou diferença significativa em relação ao déficit cognitivo $(\mathrm{F}=0,043 ; p=0,836)$ e à escolaridade $(\mathrm{F}=0,548 ; p=0,462)$. Já a ANOVA do escore no MMSE apresentou diferença significativa em relação à escolaridade $(\mathrm{F}=38,047 ; p \leq 0,000)$.

Com relação ao acesso, $95 \%$ dos sujeitos têm rádio ou televisão, $70 \%$ possuem telefone, $23 \%$ têm videocassete ou DVD e apenas $13 \%$ têm computador. Com relação ao tempo livre utilizado para participar de alguma atividade de lazer, 79\% assistem a televisão, 
$67 \%$ ouvem rádio e um único idoso informou que utiliza o computador como passatempo, para escrever, fazer cálculos ou para se comunicar. Com relação à utilidade, para $57 \%$ as TIC são um meio de se comunicar e para $52 \%$ são formas de participação do mundo. Com relação à significação da tecnologia, 30\% relacionam o termo à eficiência, à facilitação, ao progresso e à esfera da comunicação. Afirmaram que há uma evolução tecnológica se desenvolvendo, porém distante de suas vidas. O seu uso ocorre de forma incipiente, mesmo que há percepção da facilidade que poderia proporcionar em suas vidas. Alguns idosos indicaram que os jovens são os maiores beneficiados pelo uso desses equipamentos, especialmente em relação ao computador. Para eles, o descompasso no acesso às tecnologias de comunicação que ocorre entre as gerações leva a um comprometimento no âmbito relacional.

Com relação à primeira experiência, $70 \%$ indicaram que ela ocorreu em diversos ambientes, locais e situações. Para $36 \%$ a primeira experiência com a tecnologia foi com o rádio ou com a televisão. Para 30\%, o que marcou em relação à primeira experiência foi o local. Alguns afirmaram que o primeiro contato aconteceu na casa dos filhos; outros indicaram o ambiente de trabalho, e outros, ainda, indicaram locais como escolas e lojas. Um sujeito afirmou que a primeira experiência ocorreu no local de votação, isto é, o contato ocorreu em razão do procedimento adotado nas eleições brasileiras. Com relação à percepção da finalidade da tecnologia, para cerca de $10 \%$, a tecnologia é um elemento provedor de conforto e facilitação (água encanada, luz e geladeira). Para outros $10 \%$, o rádio, a televisão, o telefone e o computador são tecnologias indicadas para diversão, entretenimento e comunicação.

Com relação às percepções dos relacionamentos considerando o uso da TIC, alguns idosos descreveram que são equipamentos transformadores do convívio interpessoal, pois modificam a forma como as pessoas se relacionam. Alguns afirmaram que o acesso a equipamentos como televisão, telefone e computador lhes proporcionou uma mudança do estado emocional, resultando sentimentos como alegria, surpresa, curiosidade e expectativa. Para cerca de $20 \%$, a tecnologia é um fator negativo para o convívio interpessoal em virtude de ampliação do seu uso em detrimento dos relacionamentos. Para alguns, isso se deve à não-familiarização com os equipamentos, pois o desconhecimento cria uma elitização, afastando aqueles que conhecem daqueles que desconhecem.

\section{Conclusões}

Com relação à GDS-15, os resultados indicam que o déficit cognitivo e a escolaridade não são fatores de risco para depressão de idosos, resultados diferentes encontrados por Argimon (2004). Já ao analisar a escolaridade versus condições cognitivas por meio da aplicação do MMSE, os resultados mostraram que o desempenho nas habilidades cognitivas está diretamente vinculado ao nível de escolaridade. Resultados consistentes encontrados por Argimon (2004). A autora afirma que quanto mais anos de escolaridade o sujeito tiver maior será o fator de proteção de declínio nas habilidades cognitivas.

Com relação à utilidade das TIC, para 52\% são formas de participação do mundo. Com relação à significação da tecnologia, 30\% relacionam o termo à eficiência, à facilitação, ao progresso e à esfera da comunicação, instituindo o computador como objeto significativo da modernidade. Salomon (1992, p. 77) reforça esse argumento ao afirmar que "a tecnologia está associada a engenheiros e técnicos no sentido moderno do termo". Com relação à primeira experiência, 70\% indicaram que ela ocorreu em diversos ambientes, locais e situações. Para $36 \%$ a primeira experiência com a tecnologia foi com o rádio ou com a televisão. Para 30\%, o que marcou em relação à 
primeira experiência foi o local. Com relação à percepção da finalidade, somente para cerca de $10 \%$ as tecnologias são utilizadas para diversão, entretenimento e comunicação. Com relação às condições de acesso, para alguns a imposição do uso se deve ao interesse comum da sociedade, isto é, são objetos provedores de conforto e facilitação, tecnologias essenciais para melhorar a qualidade de vida. Para outros o acesso é voluntário, pois buscam espontaneamente aprender a utilizar os dispositivos tecnológicos que atendem às necessidades individuais de comunicação, divertimento e entretenimento. Esses resultados são corroborados pelos encontrados nas pesquisas de Peixoto e Clavairolle (2005) e Scardigli (1987). Há ainda um grupo que não fez referência alguma às tecnologias, indicando que tanto o acesso por pressão social como voluntário não ocorreu ou não é percebido, isto é, os objetos não apresentam uma dimensão normativa que manifeste ou difunda a necessidade de sua utilização. Essa dicotomia entre uma perspectiva otimista e outra pessimista em relação ao acesso as tecnologias, os resultados encontrados são confirmados nas pesquisas de Mallein e Toussaint (1987).

Com relação à percepção das tecnologias como objetos que favoreçam ou facilitam os relacionamentos, para a grande maioria a forma de utilizar esses dispositivos está impregnada de hábitos e valores. As pessoas idosas exercem o livre-arbítrio de escolher se querem ou não utilizar as novas tecnologias, mesmo quando o objeto se generaliza para toda a sociedade e o seu uso transforma o convívio. Alguns entendem que o uso da tecnologia é um fator impeditivo para o convívio interpessoal, porque o desconhecimento cria uma elitização, afastando aqueles que conhecem daqueles que desconhecem, ou, ao contrário, porque o conhecimento amplia a sua utilização em detrimento dos relacionamentos pessoais, sejam eles intergeracionais ou não. Esses resultados são confirmados pelos encontrados nas pesquisas de Barros e Goldman (1999), Caradec $(1999,2001)$ e Peixoto (2000). Por fim, mudanças do estado emocional ocorrem quando o idoso percebe que está "inserido tecnologicamente". Breton (1998) encontrou em suas pesquisas uma associação entre o uso das novas TIC e representação do prazer, estado emocional reproduzido tanto em relação às perdas quanto em relação aos ganhos da experimentação.

\section{Referências}

ALMEIDA, O. P. e ALMEIDA, S. A. Confiabilidade da versão brasileira da Escala de Depressão em Geriatria (GDS) versão reduzida. Arq. Neuropsiquiatr, v. 57, n. 2b, p. 421-426, jun. 1999.

APA. American Psychiatric Association. Diagnostic and statistical manual of mental disorders (DSM-IV). 4. ed. Washington: American Psychiatric Press, 1994.

ARGIMON, I. I. L. et al. O impacto de atividades de lazer no desenvolvimento cognitivo de idosos. Revista Brasileira de Ciências do Envelhecimento Humano, v. a, n. 1, p. 38-47, jan./jun. 2004.

BARDIN, I. Análise de conteúdo. 3. ed. Lisboa: Edições Setenta, 2004.

BARROS, M. L.; GOLDMAN, S. Bate-papo intergeracional na internet. Cadernos Pagu, v. 13, p. 37-62, 1999.

BERGE, Z. L.; COLLINS, M. P. Computer mediated communication and the online classroom: distance learning. Cresskill: Hampton Press, 1995.

BRASIL. Ministério da Saúde. Conselho Nacional de Saúde. Comissão Nacional de Ética em Pesquisa. Normas para pesquisa envolvendo seres humanos (Res. CNS 196/96 e outras). 2. ed. ampl., 1. reimpressão. Brasília: Ministério da Saúde, 2003. (Série E. Legislação de Saúde $\quad$ - $\quad$ MS). Disponível em: 
<http://dtr2001.saude.gov.br/editora/produtos/livros/pdf/03_0559_MP.pdf>. Acesso em: 26 jul. 2007.

BRETAS, M. B. A. Elementos metodológicos para a abordagem das interações telemáticas. In: FAUSTO NETO, A. et al. (Org.), Interação e sentidos no ciberespaço e na sociedade. Porto Alegre: EDIPUCRS, 2001. p. 29-48.

BRETON, P. Les personnes âgées dans le discours de promotion des nouvelles technologies: une exclusion constitutive? Prévenir, n. 35, p. 49-54, 1998.

CARADEC, V. Use of technology and ageing: an identity and family-based interpretation. In: Bouchayer, F.; Rozenkier; A. (Coord.), Technological decelopments, the dynamics of age, and ageing of the population. Paris, MIRE, 1999, p. 36-46.

. Sociologie de la vieillesse et du vieillissement. Paris: Nathan, 2001.

CASTELLS, M. A sociedade em rede. 9. ed. Trad. Roneide Venancio Mejer. São Paulo: Paz e Terra, 2006.

FOLSTEIN, M. F.; FOLSTEIN, S. E.; McHUGH, P. R. Mini Mental State: "a practical method for grading the cognitive state of patients for the clinician. Journal of Psychiatric Research, v. 12, n. 3, p. 189-198, 1975. Disponível em: < http://www.minimental.com/article.html>. Acesso em: 26 jul. 2007.

IBGE. Censo Demográfico 2000. Banco de Dados Agregados. Sistema IBGE de Recuperação Automática (SIDRA). Disponível em: <http://www.sidra.ibge.gov.br/>. Acesso em: 12 out. 2007.

KACHAR, V. A terceira idade e o computador: interação e transformações significativas. A Terceira Idade, São Paulo, v. 11, n. 19, p. 5-21, abr. 2000.

LÉVY, P. Cibercultura. Trad. Carlos Irineu Costa. São Paulo: 34, 1999.

MALLEIN, P.; TOUSSAINT, Y. L'intégration des techniques d'infonnation et de communication dans les modes de vie. Le cas du magnétoscope et de Télétel $3 \mathrm{~V}$. Culture Technique, n. 17, p. 213-219, 1987.

NERI, A. L. Velhice e qualidade de vida na mulher. In: NERI; A. L. (Org.), Desenvolvimento e envelhecimento: perspectivas biológicas, psicológicas e sociológicas. Campinas. Papirus, 2001. p. 161-200.

PARAHYBA, M. I. (1998). Evolução da mortalidade dos idosos. In: ENCONTRO NACIONAL DE ESTUDOS POPULACIONAIS, 11, 1998, Caxambu. Anais... Caxambu: ABEP.

PASCHOAL, S. M. P. Epidemiologia do envelhecimento. In: PAPALÉO NETTO, M. (Org.), Gerontologia: a velhice e o envelhecimento em visão globalizada. São Paulo: Atheneu, 2005. p. 26-43.

PEIXOTO, C. E. Avós e netos na França e no Brasil: a individualização das transmissões afetivas e materiais. In: PEIXOTO, C. E.; SINGLY, F.; CICCHELLI, V. Família e individualizaçéo. Rio de janeiro: FGV, 2000. p. 95-111.

PEIXOTO, C. E.; CLAVAIROLLE, F. Envelhecimento, politicas sociais e novas tecnologias. Rio de janeiro: FGV, 2005.

ROCHA, H. V. Representações computacionais auxiliares ao entendimento de conceitos de programação. In: VALENTE, J. A. (Org.), Computadores e conhecimento: repensando a educação. Campinas: Unicamp, 1993. Disponível em: $<$ http://www.nied.unicamp.br/publicacoes/separatas/Sep16.pdf>. Acesso em: 25 set. 2007.

ROGOFF, B.; LAVE, J. Everyday cognition: its development in social context. Cambridge: Harvard University Press, 1984.

SALOMON, J.-J. Le destin technologique. Paris: Balland, 1992.

SCARDIGLI, V. Technologies du quotidien et culture de consommation. Culture Technique, n. 17, p. 202-205, 1987. 
VALENTE, J. A. Diferentes usos do computador na educação. In: VALENTE, J. A. (Org.), Computadores e conhecimento: repensando a educação. Campinas: Unicamp, 1993. Disponível em: <http://www.nied.unicamp.br/publicacoes/separatas/Sep1.pdf>. Acesso em: 15 mar. 2007.

WHO. World Health Organization. The uses of epidemiology in the study of the elderly. Report of a WHO Scientific Group on the Epidemiology of Aging. Geneva: WHO, 1984 (Technical Report Series, n. 706).

. International Statistical Classification of Diseases and Related Health Problems

(ICD-10). 10th revision, version for 2006. Geneva: WHO/DIMDI, 1994/2006. Disponível em: <http://www3.who.int/icd/currentversion/fr-icd.htm>. Acesso em: 7 jul. 2007.

YESAVAGE, J. A. et al. Development and validation of a geriatric depression screening scale: A preliminary report. Journal of Psychiatric Research, v. 17, n. 1, p. 37-49, 1982. 